\title{
A Systematized Review of Nurses' Perceptions of Medication Errors Contributing Factors in Developing Countries
}

\author{
Abaid Mutlaq H. Al Sahli, Mohammed Ibrahim Osman Ahmed, Abdulrahman Ibrahim M. Alshammer, \\ Mohammed Nasser S. Hakami, Ismail Ahmed Hazazi, Mr. Sami Dhayaa Alqasem, \\ Fahad Sulaiman B. ALthiyabi, Fahad Nazal ALharbi, and Turky Ali Haloosh
}

\section{ABSTRACT}

Introduction: Nurses are a main factor in maintaining the safety of a patient. Nurses are the main component and are indeed at the front of dispensing medicines to patients; they also have a responsibility to maintain the patient's safety.

Objective: This systematized review intends to review studies that assess and explore nurses' attitudes and perceptions of medication errors in developing countries within the Middle East region, thereby contributing to knowledge regarding medication error risk.

Methodology: a database search has been conducted for studies related to the research question that has been formulated using PEO framework, the database includes: MEDLINE and CINAHL EBSCO and OVID, NICE, Science Direct and ERIC. The reference lists and bibliographies were also analyzed, all the studies founded during the search process were assessed according to predefine inclusion and exclusion criteria. 10 studies (6 quantitative studies, 3 qualitative and 1 which used mixed methods) were chosen and were included in this review.

Result: The studies assessed in this review were noted to have moderate to strong quality. The review found a range of factors that contribute to medication error, including both human and environmental factors. Among the contributing factors that most frequently mentioned inthis review were insufficient pharmacological knowledge, fatigue and overwork, communication breakdown, inadequate staff training, and high patient-tonurse ratio.

Conclusion and Recommendation: It would behave Saudi Arabia and neighboring countries to invest attention in this topic. Particularly advised would be additional theoretically focused research to determine the causes of medication errors.

Keywords: Nurses', Perceptions, Medication Errors, Contributing Factors.
Submitted : December 30, 2020

Published : February 22, 2021

ISSN: 2593-8339

DOI: $10.24018 /$ ejmed.2021.3.1.639

Abaid Mutlaq H. Al Sahli

King Fahad Medical City, KSA

Mohammed Ibrahim Osman Ahmed*

Erada Complex for mental health, Riyadh, KSA.

(e-mail: wadow75@gmail.com)

Abdulrahman Ibrahim M. Alshammer

Erada Complex for mental health, Riyadh, KSA.

Mohammed Nasser S. Hakami

Erada Complex for mental health, Riyadh, KSA

Ismail Ahmed Hazazi

Erada Complex for mental health, Riyadh, KSA.

Mr. Sami Dhayaa Alqasem

Erada Complex for mental health, Riyadh, KSA.

Fahad Sulaiman B. ALthiyabi

King Faisal Medical complex, KSA.

Fahad Nazal Alharbi

Erada Complex for mental health, Riyadh, KSA.

Turky Ali Haloosh

Asir health affairs, KSA

*Corresponding Author

\section{BACKGROUND}

There is a worldwide concern with regards to the safety of patient [42], [43]. Due to their role as a direct care provider, nurses are main factor in maintaining the safety of patient. The most repeated error that have occurred is medication error (MEs), which includes medicine administration error, and it is also a main factor of death and illness within hospitals [2]. Despite this focus, there is still an excessive large amount of error related to administration of medicine [3], [32]. The understanding of the reason is the solution to address and decrease medication administration errors (MEs) [26]. A nurse is recognized as the final connection within the drug therapy chain for the occurrence of the error and will typically be held responsible for errors [12]. Although, literature reviews exist regarding MEs cause [14], [23], there are no reviews addressing how nurses view MEs and the aspects contributing to MEs in in developing countries in
Middle eastern regions. Therefore, this systematized review endeavours to evaluate available data regarding the causes and contributing aspects of MEs as determined by the nurses themselves in KSA hospital. The review for the current topic is highly motivated by a serious amount of errors that have occurred in the Kingdom of Saudi Arabia (KSA) where the author has previous held an occupation, as well as this being an essential topic on a national level. There is no definitive understanding on the aggregate MEs within the KSA. Despite this, the first approach is to ensure the solution is placed upon the nurse's perception and predisposing factor [24].

There are no possibilities of determining the amount of MEs within the third world and developing countries. This is due to the limited amount of archiving and reporting systems and the data registration system is not present. There are, however, a high amount of complaints received from medical staff to courts along with increased judiciary evidence; there are professionals [18] that would deem MEs to be a large issue within the aforementioned countries .Although it would 
have been most beneficial to conduct a direct review of studies performed in the KSA context, the limited number of available research papers in this topic within KSA made the author to take a wider position by including studies from middle eastern countries neighboring to KSA that have similar working condition and shared with the KSA the language and culture and religion.

\section{A. Leading Factors in Medication Errors}

MEs is not limited to individual factors such as not having a full knowledge. There are other factors that contribute to ME such as system and environment aspects [38]. There are typically two types of drugs that are dispensed in hospital; stock based and unit-dose dispensing system. 'Stock based' is when the supply of medicines is stored within wards and are being dispensed by the nurse in accordance with the prescription by the doctors. There is a higher amount of MEs through this method. Unit-dose dispensing system is when the drugs are dispensed in accordance with the individuals' requirements for the duration of 24hours. There are less MEs through this method [41].

A study conducted by [11] regarding nurses 'viewpoint of MEs and their approach to reports. The most significant cause of $\mathrm{ME}$ is there is a failure of patient identification at $35 \%$. The second most significant is tiredness and exhaustion at $25 \%$. The study has pointed out that most nurses do not report MEs because of concerns with consequences from co-workers and managers. A survey conducted by [39] with 983 participants show that the first three errors are due to misunderstanding of doctors' handwriting, distraction, and exhaustion.

Two studies with regards to MEs were conducted in Jordan. [35] have utilized the Modified Gladstone's scale for data collection purposes in terms of MEs' source and reporting as well as the rate. The study's data show that the major causes of MEs from nurses are due to the following starting from most to the least; inferior quality or damaged packaging/labels of drugs, confused one medicine with another as well as its functions and infusion devices, distraction by patients, co-workers or events within the environment, struggling to deploy infusion devices, fatigues, failure to verify patients' name, wrong prescriptions, medicine confusion, illegibility of prescriptions' texts and miscalculation of dose [6] have conducted the second study where 200 nurses were surveyed spread across 3 hospitals with regards to MEs in accordance with their view point. The study's data show that the major causes of MEs are due to the following starting from most to the least; excessive workload (41\%), newly employed (20\%), neglect (15\%), insufficient medicine knowledge (11.9\%), lack of training (4.8\%), convoluted prescription $(4.8 \%)$ and lack of knowledge with regards to patient's condition $(1.6 \%)$.

\section{B. Impact and Scale of MEs}

There are various causes of MEs and it is not fixed on one specific cause; the findings of a generic cause is improbably as each study have too many variables, populations, measurements and methods and as such MEs would not be observed [32]. However, developed and developing countries are experiencing MEs as a frequent issue and reports have found that there are various aspects of causes such as knowledge and experience of health workers [14] MEs have posed a main obstacles due to its extent in various hospital environment. There are $10 \%$ MEs reported for patients that have been admitted to hospital as intensive care unit with serious magnitudes [35]. There is a large percentage of MEs thus this shows that it is essential to minimize preventable errors in order to improve patientcare [34]. MEs are mainly result from iatrogenic measures. There is a possibility of the MEs to formed into extended stay in hospital, morbidity, preventable diagnosis tests, preventable deaths and treatments [30]. MEs are related to administration of medicine that could have been avoided if there is a presence of efficient system of control [28], [31]. Morbidity and mortality as a result of MEs have been analyzed by researchers within the hospitalized patients for the duration of 3 years in USA on a case-control basis (ADR). There has been a strong rise in terms of hospital costs and further length of stay from $\$ 677$ to $\$ 9,022$. There is a rise of patient mortality rate with odds ratio being 1.88 (1.3-3.7) and post-discharge disability [49]. MEs are viewed as a primary health concern in public. This is due to the hurt resulted from them, affecting many parties such as patients, families and healthcare providers. Nurses are the main component and are at the front of dispensing medicines to patients and also have responsibility to the patient's safety; therefore, they are well versed with the problems as well as being significant in terms of formulating plans and strategies to minimize the problems and to establish a developed health system (Nurses have the necessary knowledge as well as their established relationship with the patients [13]. This means that they are in a prime position that could determine and avoid the preventable errors. [45]. have suggested that there is a requirement to ensure all MEs' causes are known through querying nurses' viewpoint. The nurses should know the source of the error and the associated risk aspects of MEs.

\section{Objectives}

-To systematically review literature about medication errors in developing countries within the middle Easter region.

- To assess the available literature in terms strength and weakness of their methodology.

- To review studies that investigate the experiences and perceptions of nurses in order to determine which aspects may increase medication error risk.

\section{Introduction}

The systematized literature review presented as this dissertation examines the contributing factors leading to medication errors by nurses working in developing Middle Eastern countries. This chapter will provide an overview of the selected methodologies that employed throughout this dissertation, beginning with description of the unique properties of a systematized review as compared to systematic review. Secondly, this chapter will explain the process that was employed for the entirety of this review.

\section{E. Systematized Literature Reviews}

A systematized literature review contains elements of a systematic literature review but does not recognize the output as systematic [24]. It is recognized as the most frequently utilized review type and is widely seen as an excellent means of summarizing information regarding a certain topic that has been published [24]. Therefore, to understand a systematized 
literature review framework used in this dissertation. The definition of systematic review should be clarified. A systematic review is an intentionally planned process that utilizes explicit methodology in order to examine published works in a critical manner and to methodologically select appropriate works to review [27]. The rigorous methodology means the articles addressed in a systematic literature review can be deemed original work [27]. When providing information to inform professional practice in the medical sciences, it is vital that such information is founded in strong evidence [16]. The two review types previously described are founded in current evidence, although follow different construction methods [16]. The evidence-based pyramid, which ranks review methodologies according to evidence, ranks systematic reviews at second highest with a level 8 [25]. Cochrane systematic reviews are ranked in the highest position [19]. These reviews examine primary healthcare research that has been carried out by the Cochrane Collaboration, an internationally recognized research network [27].

\section{F. Search Strategy Steps}

The systematized review has undertaken the following overlapping process that include question formulation, systematic identification of eligible studies, systematic selection of included studies, study quality evaluation, data collection and gathering, findings analysis, and composition of the dissertation.

Question Formulation: In developing countries in the Middle East region, what are the contributing factors towards nurse medication error?

Systematically Looking for Appropriate Studies: This review begins with Google Scholar, used to establish a general view of the topic. The outcomes were employed to examine the titles, abstracts and index terms to create a wider collection of search terms, which, in turn, could be employed for the larger search in the second stage, involving all databases used in the study.

The second stage involves an examination of all peer reviewed, published studies looking into medication errors made in nursing contexts, restricting results to those published in the last 16 years only, this period was chosen to ensure the results are up to date enough and also because of the major national safety changes resulting from the 2000 Institute of Medicine in America (IOM) report entitled, 'To Building a Safer Health System [4], [6].

The CINAHL and Medline databases were examined, which include the majority of nursing studies [15], together with EBSCO and OVID, NICE (National Institute for Health and Care Excellence), Science Direct and ERIC (Education Resources Information Centre), in order to create more detailed and thorough results. Non-automated analyses of journals like the American Journal of Nursing, British Nursing Index were also undertaken under the scope of this review. These manual searches involve an examination of all volumes of specific journals to pinpoint publications which might not be indexed in databases, are incorrectly indexed, are editor letters with research results included, or are transcripts of conferences.

Lastly, the reference lists and bibliographies of all selected papers are analyzed, which is beneficial when it comes to finding studies not highlighted in the mother search stages, even though this takes a very long time to complete. On the other hand, this stage of searching is made more confusing by the substantial number of citation mistakes in journal articles, which have been shown to have an error in up to $45 \%$ of cases [15] state that suitable research keywords and terms must be highlighted prior to searching commencing. In line with the PEO framework, this search's keywords are presented in the table below.

Selection of Studies: When it comes to systematic reviews, the study population consists of earlier primary research surrounding the topic at hand, which will be chosen based on the eligibility criteria of the review protocol. These inclusion criteria allow the review question to be put into action through the review question by presenting its components in a way that is suitable for the selection process. These criteria allow the review to not suffer from bias, where the researcher might make study selections based on their results [43]. The systematized review of this dissertation undertook a similar process, although the criteria were created and applied after the search process; the author's increased understanding of the literature allowed for a more appropriate set of criteria.

\section{G. Inclusion Criteria}

If a study fulfilled all of the following criteria it was considered eligible for the review:

Primary research studies attempting to:

- Examine medication errors from the perspective of nurse.

- Examine medication error cause.

- Look into the reasons behind medication errors across various stages of the management process in the context of a community or hospital-based mental healthcare service.

- English or Arabic language papers.

- Peer reviewed, published literature only.

- Published in the last ten years, in order to ensure a modern view and take into account the extensive national safety changes coming after the 2000 Institute of Medicine (IOM) report 'To Building a Safer Health System'.

\section{H. Exclusion Criteria}

If a study fulfilled any of the following criteria it was excluded:

- Primary research studies examining topics other than nurse or medication errors.

- Papers looking into medication errors from a perspective other than that of nurses, or from reviewing the hospital or prescription files.

- Reviews, opinion papers, letters and editorials (nonprimary studies).

- Studies languages other than English or Arabic. Papers which are not reviewed yet.

- Studies published earlier than 2004.

\section{Literature Review}

Following the search of electronic databases, there were 750 studies found, which then needed to undergo a threestage appraisal process in order to reach the relevant data base studies. Firstly, the title and abstract were evaluated, where duplicate studies and those with titles or abstracts unrelated to the topic of interest were discarded. This brought the 
number of studies down to $40(n=40)$. Following this, the full text was appraised and examined with regard to its examination of the perception and experience of nurses with regards to medication errors. An evaluation of these research papers is also completed relative to their suitability as dictated by the exclusion and inclusion criteria shown in Table 1 . Following these stages, there were $14(n=14)$ relevant articles remaining.

The third stage involved a deeper review of these 14 articles in order to ascertain which had sufficient detail on the topic of interest, 4 studies were concern about the incidence of the MEs by reviewing hospital records. Overall, 10 studies (6 quantitative studies, 3 qualitative and 1 which used mixed methods) were chosen and are involved in the deeper examination of the document Table 3 . This is low number of research paper indicative of the relatively low amount of research conducted on this topic in developing countries within the Middle Eastern region. Of note is that the majority of studies were published after 2006, reflecting an uptick in interest and awareness of the topic within the past decade.

\section{J. Population and Samples}

The studies consulted cover a total population of 65 experts in medication safety [9], 4,070 nurses and 38 observed patients in Saudi Arabia 45. Sample sizes examined ranged from 38 to $2000(45$,$) . Five studies reported response rates,$ which was recorded between $46.6 \%$ and $100 \%$ [5], [37] respectively. Only one piece of research explained that the sample size was determined according to statistical power [5]. All studies examined explicated the demographic characteristics of the population examined. The average participant was a female with a bachelor's degree and at least 5 years of working experience. The high percentage of female nurses, ranging from $46 \%$ [34] to $87.2 \%$ [20], could impact result validity by introducing gender bias. However, in KSA and other middle eastern countries the female nurses represent the largest portion of the nurses compared to male nurses, so it is expected to include more female nurses in this study [19]. All studies consulted accurately outlined the methodology employed for sampling. Six studies used the convenience sampling method, two used the random selection sampling method [18], and one used the purposive sampling method [9].

\section{K. Data Collection Instruments}

The quantitative studies included in this review are united in their use of self-completion questionnaire surveys as a data collection instrument. Surveys are advantageous in that the yallow for flexibility and broad scope of coverage. The ability to reach a significant proportion of the study population is particularly important in this context as there is limited knowledge on the topic of MEs in developing countries within Middle Eastern region according to nurse perspective. The broad reach of a survey will contribute to basic comprehension of the topic at hand. It should be noted that the external validity of the aforementioned studies may be called into question as the self-completion methodology may mean some responses were incorrectly interpreted [26]. This may mean that some outcomes, particularly those describing beliefs and feelings, may be likewise inaccurate [26]. Of the eight studies that employed questionnaires, only one utilized a previously-developed questionnaire. [34] employed the
Modified Gladstone's scale to measure nurses' perception regarding factors resulting in medication error (over $50 \%$ of questions), the rate of medication errors as reported to nurses, and nurses' opinions regarding medication error reporting [37].

\section{Data Analysis}

Descriptive and inferential statistical analysis is employed in seven of the studies addressed in this review. one studies [6] only apply statistical analysis, whiles one study [9] analyses qualitative data using content analysis [25].

Incidence and the Most Common Type of Medication Error According to the Reviewed Studies Statistics are presented by two reviewed studies concerning the prevalence of medication errors amid participants. It was established 18 that $64.55 \%$ of the nurses had encounters of errors in medication during the 3 months of the research. The average amount of medication errors made by every nurse in the 3 month time frame of the research was 7.4. In Jordan, [34] documented a minimum of $42.1 \%$ of the participant as having made one medication error for 3 months. The average number of errors for every nurse was computed as 2.2.3 researches offer a description of the most widespread kind of medication error. [6] states that the highest percentage of medication errors comprised when medication was administered to the incorrect patient $(26.2 \%$ of all the medication errors) succeeded by $22.2 \%$ for incorrect dosage. Nonetheless, frequency and changing of medication comprised the least frequent, with $3.1 \%$ and $1.5 \%$ of medication errors correspondingly. The greatest extents of medication errors $(48.4 \%)$ took place when the medication was administered to an individual by a nurse in various phases such as patient observation $(31.7 \%)$, dispensation of drugs $(11.1 \%)$ as well as omission of medication $(5.6 \%)$. Alternative extents of medication errors took place when ordering the medication by a physician $(27.0 \%)$ and by the pharmacist $(24.6 \%)$, during transcribing of medication $(15.9 \%)$ as well as administering $(8.7 \%)$. Alternative extents of medication errors took place while ordering of the drugs by a physician $(27.0 \%)$ as well as by the pharmacist $(24.6 \%)$, within medication transcribing $(15.9 \%)$ as well as dispensation $(8.7 \%)$. The most widespread kinds of medication errors as stated by [18], [20] comprised wrong dosage and rate of infusion.

\section{The Predisposing Factors for MEs According to Reviewed Studies Included in this Dissertation}

Three studies carried out in KSA describe aspects that lead to increased risk of Saudi nurse medication error. These studies also provide a review of Saudi expert opinions on the topic of medication administration safety. Additional studies carried out in nearby countries that host a relatively similar working environment as well as similar nursing preparatory education were also included in this review with the intention of providing a broad view of contributing factors. The inclusion of neighbouring countries not only allows for examination of further factors, but also compensates for the low number of studies available within Saudi Arabia. Regardless of location, the studies examined have significant overlap in their findings regarding contributing factors towards medication error. Research conducted by [5] utilizes correlational, descriptive and cross-sectional design to 
examine the various aspects related to errors in nurse medication administration. The research investigates two regional KSA hospitals and 209 nurses, as well as 288 hospital records describing medication error. The outcomes of the research demonstrate that nurses attribute errors mainly to physician communication and workload. Additional contributing factors cited include interruption during administration duties in favour of other tasks and nursing shortages. Factors that were noted as having a low level of influence regarding medical error include the use of abbreviations, verbal orders and medication packaging. AlSaleh and Ramadan found similar results in their 2012 research, which polled medical staff on their opinions regarding medical error contributing factors using a 50questionquestionnaire. The questionnaire received 932 unique responses that noted major contributing factors to be lack of education or experience, distraction, inadequate resource coordination, heavy workload, time constraints, and fatigue. According to both of these studies [5], [6], medical staff are susceptible to medication error due to workplace contexts that are not optimally designed to support safety measures. Another study utilized round-table discussion to review the contributing factors and flaws in KSA medication safety practice [9]. This research consulted 65 experts, including 14 hospital pharmacists, 12 clinical pharmacists, nine hospital quality coordinators/medication safety officers, six physicians, five pharmacy directors, six academics, three community pharmacists, three health authorities, three nurses, two pharmacy students and two pharmaceutical industry representatives. The findings were in line with the previous studies addressed [5], [6] in that workload and inadequate human resources were cited as the major contributing factors towards medication error. In addition, the study carried out by [9] found communication gaps, inadequate hospital medication safety programmes, and minimal technology use to be further contributing factors towards the occurrence of medication error. The same study identified further factors, including inadequate policies and procedures, insufficient human resources (including medication safety officers), and inappropriate medication labels. Some participants in the round table discussions pointed to multilingualism and cultural diversity among healthcare professionals as potential issues, particularly as verbal orders and communication are so vital when it comes to decreasing the occurrence of medication error [39] carried out a similar piece of research in the nearby UAE. Their research relied on a questionnaire distributed to 170 nurses employed by Ajman's Gulf Medical Hospital to assess medication error knowledge and attitudes. The majority of the nurses surveyed reported strong agreement that refresher courses on medication safety $(64.1 \%)$ and medication administration $(53.5 \%)$ provided to nurses have the potential to significantly reduce medication errors. Study participants also noted that a medication assignment system and the ability to seek physician clarification are addition means to reduce medication error. The first study to examine medication error among nurses in Jordan was conducted by [34]. Using a modified Gladstone's scale [33], [36], the study evaluated 799 nurses across 24 hospitals and found the top three perceived contributing factors towards medication error to be confusion among nurses regarding the function and kind of infusion devices, poor quality or damaged packaging/labels, and distraction caused by co-workers, patients or unit events. Unlike the previously reviewed research [5], [37] did not determine education level or workload to be potential factors. This is due to the fact that the original Gladstone's scale of medication error does not cover all factors. Following on [34] study, in [6] conducted similar research surveying 200 Jordanian nurses on the types, stages and issues they perceived as contributing towards medication error. Of the 200 registered nurses surveyed, 126 completed the questionnaire and reported insufficient training, heavy workload, and minimal experience or unfamiliarity of new hires with medication as contributing factors. In Iran, [35] carried out a cross-sectional study on nurse perceptions of medication error in 2009. The researchers randomly selected 237 nurses employed at Imam Khomeini Hospital to complete a 17-question survey regarding medication error and demographic information. The study found that use of abbreviations in prescriptions and drug name similarities were the most common factors contributing towards medication error. The study also determined there were some managerial and human contributing factors including insufficient staff training, heavy workload, and inadequate pharmacological knowledge. A quantitative study conducted by [20] examined medication error specifically in the emergency department. This piece of research focused on 94 nurses employed at the Khomeini Hospital Complex and determined that medication errors are a major risk among the emergency department's nursing staff. The research determined the most dominant causes of medication error in this context were organization and human in nature, and included insufficient pharmacological knowledge, use of abbreviated names, high patient-to-nurse ratio, and hard work-induced fatigue. [20] carried out their research in Iranian hospitals, surveying 239 randomly selected nurses and head nurses regarding contributing factors towards medication error. The participants noted individual, managerial, environmental, equipment-related, patient-related, professional and team factors among the medication error causes. Significantly, head nurses 'responses correlated only with managerial, team and patient-related factors. However, the study did not clearly describe what these topics of errors are meant to include. Another Iranian study was carried out by [42] in an 11bedinternal ICU university hospital. The prospective observational study employed trained pharmacists to record all processes performed on a selected patient throughout the course of each shift, and eventually covered 38 shifts. The study documented 578 opportunities for error and 442 errors. The errors included administration errors $(9.8 \%)$, prescribing errors $(6.8 \%)$, transcription errors $(2.2 \%)$ and dispensing errors $(2.3 \%)$. The major factors cited as cause of these errors included rule violation, insufficient pharmaceutical knowledge, and memory lapse. Although the observation method used in this research is more effective than the other pieces of research assessed, the rate and cause of medical error may be underestimated.

To summarize, the research assessed found general agreement in terms of causes and contributing factors towards medication error. Frequently cited factors among KSA and neighboring country hospitals include heavy workload, 
insufficient pharmacological knowledge, large variety of medications available, insufficient human resources, poor communication among hospital staff, and use of abbreviated names.

\section{N. Discussion and Limitation of the Review}

Globally, research reports that medication errors are liable to occur at any stage throughout the process of medication management. Clinicians in Saudi Arabia are thus likewise concerned about medication error [37] That said, relatively few studies on this topic have been carried out in hospitals or primary care contexts in the country (A. E. [5], [9]).

The incidence of the MEs encompassed within the appraised researched studies varied between $42.1 \%$ and $64.55 \%$, nonetheless this range was only reliant on 2 research while the rest encompassed studies which did not disclose the occurrence rate of MEs amid the participants, or not aimed at scrutinizing the occurrence of MEs. Subject to the results from these two researches, it would appear that the occurrence of medication error amid developing nations from the middle eastern area were slightly higher compared to those documented for some developing nations, for instance [37] carried out a research within Danish hospitals and discovered the level of nursing medication mistakes (43\%) to be reduced in contrast to what [18] established and comparable to [34]. This could mirror the occurrence of MEs within the area of concern, nonetheless more research is required spanning various nations to accurately appraise the precise occurrence of the MEs. Being aware of the occurrence as well as the most common type of MEs assists in forming strategies amid the nations to lessen the matter of MEs and assist in planning tactics. Research by [3] highlighted that the individual most responsible for the incidence of errors of medication is the nurse, (i.e., while administering the drugs to an individual), seconded by a physician (i.e., while ordering the medication), and ultimately, a pharmacist (i.e., within transcribing and dispensation of medication). Similar results were disclosed within the literatures. In comparison, several researches highlighted that physicians had committed the greatest amount of medication errors seconded by pharmacists and subsequently nurses. The study by [3] highlighted that selecting the incorrect patient or the incorrect dosage comprised the two chief types of medication errors. Comparable results are additionally disclosed [35]. Unsuitable dosage and rate of infusion comprised the most widespread kinds of documented medication errors as stated by [18], [20]. The most significant errors were related to intravenous infusions $(60.78 \%)$. appraised medication errors within a pediatric hospital and discovered $61 \%$ of the errors to be associated with intravenous injections. Even though medication errors may be caused using any drug, the pharmacological features or disproportionate employment of several of the classes of drugs raise the danger of errors. Complications of errors within intravenous injections are more contrasted to alternative techniques of drug dispensation. There have additionally been reports regarding the occurrence of death and grave injuries after errors in intravenous injection (encompassing incorrect drugs, dosage or dilution). As stated by [36], this comprised significant consequences associated with the objective of this dissertation, as these kinds of medication errors require particular attention and training schemes in order to prevent their incidence and eradicate their associated danger. Limited human and physical resources mean healthcare providers are unable to appropriately delegate tasks. One of the included studies found that the lack of resources and limited uses of technology increase the occurrence of MEs [9]. Hospital administrators would benefit from addressing this situation in their budgeting and recruitment processes, although some middle eastern countries have a nation-wide nursing shortage [8]. Policy makers should take this into consideration and should recognize the potential of additional staff to reduce the instance of adverse events and loss of life, despite the higher costs involved in their recruitment [5]. Without additional staffing, this issue is unlikely to be resolved, as nurses must continue to prioritize patient safety within the systemimposed time and work constructions. Training programs and continuous professional development courses among nurses in the Middle Easter region could help in providing the nurses with the knowledge and the skills that help in reducing the MEs. Medication errors are widely acknowledged to be frequently caused by lack of pharmacological knowledge This situation may be a result of inadequate clinical pharmacology instruction [14]. This is particularly true of nurse knowledge regarding "high-risk" groups of medication, such as those used to treat cardiovascular disease. This is significant given the link between such medication and medication error [38]. He reviewed studies also pointed towards illegible handwriting as a cause of medication error. Some study participants pointed to multilingualism and cultural diversity as causes for such misunderstandings in communication [34], [37]. One local researcher found that the majority $(72 \%)$ of Saudi Arabian physicians wrote lowquality prescriptions [39]. As a means to reduce the errors resulting from illegible handwriting, some Saudi researchers have promoted computerized provider order entry (CPOE) use [9]. Research has shown that correctly implemented CPOE can positively reduce medication error.

\section{REVIEW LIMITATIONS}

The systematic review described in this dissertation is subject to several limitations. The search range was regulated in accordance with time available, which meant the intention exclusion of grey literature and unpublished works. In addition, the search was restricted to articles published in either English or Arabic, excluding works published in other languages.

These limitations are due to the systematic design of the review and as well to the available resources at the time of the review. In particular, human resources were limited, as this review was carried out by only one researcher [22]. The selection criteria employed in this review is also subject to limitations. These include a limited number of searched databases and keywords, and a limited geographic focus. It is possible that some research may have been published in an unaddressed journal, or in the context of another country outside of KSA and its neighbours. Despite a methodological manual and electronic search process, there is a possibility some related studies were not uncovered. There is also a possibility that a different researcher would have employed different criteria or selected different research for review. 
Regardless of the above limitations, it is still possible to meditate on the various pros and cons of the methodology employed for this dissertation. The review guidance can be found in the comprehensive and meticulous description of the search methods, and as well in the comprehensive and meticulous discussion of the recommendations drawn from the review outcomes. Although research bias was accounted for using a wide-ranging approach, it is possible a different researcher or group of researchers would have come to different conclusions. For this reason, future empirical research that further expands knowledge on this topic would be beneficial.

\section{CONCLUSION:}

Based on the findings of this review, it would be hove Saudi Arabia and neighbouring countries to invest attention in this topic. Particularly advised would be additional theoretically focused research to determine the causes of medication errors. It would be advisable for such research to emphasize mitigation strategies in order to impact patient safety over the long term. Going forward, the concerns described in this review should be taken into consideration when designing initiatives aimed at reducing medication error. This review has also noted a need for additional education, research and interventions aimed at guaranteeing safe use of medications.

\section{RECOMMENDATIONS:}

- It is important to improve the working conditions for nurses. In particular, a lightened workload and reduced hours would have beneficial impact.

- Continuous education programmes and competency assessments could be implanted, and nurses should be encouraged to participate in such programmes and assessments.

- Healthcare professionals would benefit from on-going education and competency assessment that emphasise medication safety.

\section{ACKNOWLEDGEMENTS}

My deep gratitude is expressed here to my supervisor Dr Emma Gillaspy, for agreeing to work with me and for the time and commitment which she gave, along with all of the detailed advice, feedback and guidance, which was essential in completing this thesis. I also wish to thank the head of programme, Dr Michelle Howarth, Dr Jackie Leigh, the leading education in academic and practice, Dr Angela Cotton (research methodology) and Mrs Lyn Rosen whose help was also essential in making this thesis a reality. Many thanks to you for your generous efforts and guidance.

\section{REFERENCES}

[1] Acharya, A. S., Prakash, A., Saxena, P., \& Nigam, A. (2013). Sampling: Why and how of it. Indian Journal of Medical Specialties, 4(2), 330-333.

[2] Adams, M. P., \& Carol Urban PhD, R. N. (2015). Pharmacology: Connections to nursing practice: Prentice Hall.

[3] Agency for Healthcare Research and Quality (AHRQ). (2013). Making Health Care Safer II: an updated critical analysis of the evidence for patient safety practices. Retrieved from Evidence report/Technology assessment number 211.AHRQ, Rockville, MD.

[4] Al-Faris, E. A. (1999). The prevalence of abnormal results of annual investigations among diabetic patients with different risk factors. Annals of Saudi medicine, 20(3-4), 206- 210.

[5] Al-Saleh, K. S., \& Ramadan, M. Z. (2012). Studying Medical Errors among Hospital-Staff at Saudi Health Providers. Journal of Materials Science and Engineering, 2(1), 43-54.

[6] Al-Shara, M. (2011). Factors contributing to medication errors in Jordan: a nursing perspective. Iranian journal of nursing and midwifery research, $16(2)$.

[7] Alahmadi, H. A. (2010). Assessment of patient safety culture in Saudi Arabian hospitals. Quality and Safety in Health Care, 19(5), 1-5.

[8] Alghamdi, M. G., \& Urden, L. D. (2016). Transforming the nursing profession in Saudi Arabia. Journal of nursing management, 24(1), E95-E100.

[9] Aljadhey, H., Mahmoud, M. A., Hassali, M. A., Alrasheedy, A., Alahmad, A., Saleem, F., Almalki, M., FitzGerald, G., \& Clark, M. (2011). The nursing profession in Saudi Arabia: An overview. International Nursing Review, 58(3), 304-311.

[10] American Society of Hospital Pharmacists. (1982). Council on clinical affairs: ASHP standard definition of medication error. Retrieved from American Journal of Hospital Pharmacy:

[11] Armitage, G. (2009). Human error theory: relevance to nurse management. Journal of nursing management, 17(2), 193-202. 60.

[12] Armitage, G., \& Knapman, H. (2003). Adverse events in drug administration: a literature review. Journal of nursing management, 11(2), 130-140.

[13] Balas, M. C., Scott, L. D., \& Rogers, A. E. (2004). The prevalence and nature of errors and near errors reported by hospital staff nurses. Applied Nursing Research, 17(4), 224230 .

[14] Brady, A., Malone, A., \& Fleming, S. (2009). A literature review of the individual and systems factors that contribute to medication errors in nursing practice. Journal of nursing management, 17(6), 679-697.

[15] Brazier, H., \& Begley, C. M. (1996). Selecting a database for literature searches in nursing: MEDLINE or CINAHL? Journal of Advanced Nursing, 24(4), 868-875.

[16] Brettle, A., \& Grant, M. J. (2004). Finding the evidence for practice: a workbook for health professionals: Churchill Livingstone.

[17] Buysse, V., Winton, P. J., Rous, B., Epstein, D. J., \& Lim, C. I. (2012). Evidence-based practice. Zero Three, 32, 25-29.

[18] Aboshaiqah, A. E. (2014). Nurses' Perception of Medication Administration Errors. American Journal of Nursing Research, 2(4), 63-67.

[19] Carlton, G., \& Blegen, M. A. (2006). Medication-related errors: a literature review of incidence and antecedents. Annual review of nursing research, 24(1), 19-38.

[20] Cheragi, M. A., Manoocheri, H., Mohammadnejad, E., \& Ehsani, S. R. (2014). Types and causes of medication errors from nurse's viewpoint. Iranian journal of nursing and midwifery research, 18(3).

[21] Clarke, B., Gillies, D., Illari, P., Russo, F., \& Williamson, J. (2014). Mechanisms and the evidence hierarchy. Topoi, 33(2), 339-360.

[22] Dwan, K., Altman, D. G., Arnaiz, J. A., Bloom, J., Chan, A.W., Cronin, E., Gamble, C. (2008). Systematic review of the 
empirical evidence of study publication bias and outcome reporting bias.

[23] Ehsani, S. R., Cheraghi, M. A., Nejati, A., Salari, A., Esmaeilpoor, A. H., \& Nejad, E. M. (2013). Medication errors of nurses in the emergency department. Journal of medical ethics and history of medicine, 6 .

[24] Freeman, R., McKee, S., Lee-Lehner, B., \& Pesenecker, J. (2013). Reducing interruptions to improve medication safety. Journal of nursing care quality, 28(2), 176-185.

[25] Fry, M. M., \& Dacey, C. (2007). Factors contributing to incidents in medicine administration. Part 1. British journal of nursing, 16(9).

[26] Grant, M. J., \& Booth, A. (2009). A typology of reviews: an analysis of 14 review types and associated methodologies. Health Information \& Libraries Journal, 26(2), 91-108.

[27] Groves, R. M., Fowler Jr, F. J., Couper, M. P., Lepkowski, J. M., Singer, E., \& Tourangeau, R. (2009). Survey methodology (Vol. 561): John Wiley \& Sons.

[28] Guyatt, G., Rennie, D., Meade, M. O., \& Cook, D. J. (2002). Users' guides to the medical literature: a manual for evidencebased clinical practice (Vol. 706): AMA press Chicago, IL.

[29] Hartnell, N., MacKinnon, N., Sketris, I., \& Fleming, M. (2012). Identifying, understanding and overcoming barriers to medication error reporting in hospitals: a focus group study. BMJ quality \& safety, 21(5), 361-368.

[30] Higgins, J. P. T., \& Green, S. (2008). Cochrane handbook for systematic reviews of interventions (Vol. 5): Wiley Online Library.

[31] Hillestad, R., Bigelow, J., Bower, A., Girosi, F., Meili, R., Scoville, R., \& Taylor, R. (2005). Can electronic medical record systems transform health care? Potential health benefits, savings, and costs. Health Affairs, 24(5), 1103-1117.

[32] Hosseinzadeh, M., Ezate Aghajari, P., \& Mahdavi, N. (2012). Reasons of nurses' medication errors and persepectives of nurses on barriers of error reporting. Hayat, 18(2), 66- 75.

[33] Hussain, E., \& Kao, E. (2005). Medication safety and transfusion errors in the ICU and beyond. Critical care clinics, 21(1), 91-110.

[34] Johnson, J., \& Thomas, M. (2013). Medication errors: Knowledge and attitude of nurses in Ajman, UAE. Reviews of Progress, 1(4), 2321-3485.

[35] Kanjanarat, P., Winterstein, A. G., Johns, T. E., Hatton, R. C., Gonzalez-Rothi, R., \& Segal, R. (2003). Nature of preventable adverse drug events in hospitals: a literature review. American Journal of Health System Pharmacy, 60(17), 1750-1759.

[36] Keers, R. N., Williams, S. D., Cooke, J., \& Ashcroft, D. M. (2013a). Causes of medication administration errors in hospitals: a systematic review of quantitative and qualitative evidence. Drug safety, 36(11), 1045-1067.

[37] Keers, R. N., Williams, S. D., Cooke, J., \& Ashcroft, D. M. (2013b). Prevalence and nature of medication administration errors in health care settings: a systematic review of direct observational evidence. Annals of Pharmacotherapy, 47(2), 237-256.

[38] Lisby, M., Nielsen, L. P., \& Mainz, J. (2005). Errors in the medication process: frequency, type, and potential clinical consequences. International Journal for Quality in Health Care, 17(1), 15-22.

[39] Mayo, A. M., \& Duncan, D. (2004). Nurse perceptions of medication errors: what we need to know for patient safety. Journal of nursing care quality, 19(3), 209-217.

[40] Moyen, E., Camiré, E., \& Stelfox, H. T. (2008). Clinical review: medication errors in critical care. Crit Care, 12(2), 208.

[41] Mrayyan, M. T., Shishani, K., \& Al-Faouri, I. (2007). Rate, causes and reporting of medication errors in Jordan: nurses' perspectives. Journal of nursing management, 15(6), 659-670.

[42] Mueller, S. K., Sponsler, K. C., Kripalani, S., \& Schnipper, J. L. (2012). Hospital-based medication reconciliation practices: a systematic review. Archives of internal medicine, 172(14), 1057-1069.
[43] National Collaborating Centre for, M., \& Tools. (2008) Quality Assessment Tool for Quantitative Studies: McMaster University Hamilton, ON.

[44] Neyaz, Y., Qureshi, N. A., Khoja, T., Magzoub, M., Haycox, A., \& Walley, T. (2011). Physicians' edication prescribing in primary care in Riyadh city, Saudi Arabia.Literature review, part 1: ariations in drug prescribing. Eastern Mediterranean health journal, 17(2), 126.

[45] Ong, W. M., \& Subasyini, S. (2013). Medication errors in intravenous drug preparation and administration. Med J Malaysia, 68(1), 52-57.

[46] Pham, J. C., Aswani, M. S., Rosen, M., Lee, H., Huddle, M., Weeks, K., \& Pronovost, P. J. (2012). Reducing medical errors and adverse events. Annual review of medicine, 63, 447-463.

[47] Taylor, D. (1999). Introduction to Research Methods medicine, $319,1618$.

[48] Taylor, M. K. (1998). The practical effects of errors in reference lists in nursing research journals. Nursing research, 47(5), 300-303.

[49] Sinnemäki, J., Sihvo, S., Isojärvi, J., Blom, M., Airaksinen, M., \& Mäntylä, A. (2013). Automated dose dispensing service for primary healthcare patients: a systematic review. Systematic reviews, 2(1), 1 .

[50] Vincent, C., Burnett, S., \& Carthey, J. (2013). The measurement and monitoring of safety. The Health Foundation.

[51] Vazin, A., \& Delfani, S. (2012). Medication errors in an internal intensive care unit of a large teaching hospital: a direct observation study. Acta Medica Iranica, 50(6), 425.

[52] World Health, O. (2008). World Alliance for Patient Safety Progress Report 2006-2007.

[53] Wagner, A. K., Chan, K. A., Dashevsky, I., Raebel, M. A., Andrade, S. E., Lafata, J. E., Platt, R. (2006). FDA drug prescribing warnings: is the black box half empty or half full? Pharmacoepidemiology and drug safety, 15(6), 369-386.

[54] http://apps.who.int/iris/bitstream/10665/75169/1/WHO_IER_ PSP_2008.03_eng.pdf. 